\title{
Generalizations of the Nevanlinna-Pick interpolation Problem
}

\author{
Minyue Fu and Kaushik Mahata
}

\begin{abstract}
This paper aims at generalizing the well-known Nevanlinna-Pick interpolation problem by considering additional constraints. The first type of constraints we consider requires the interpolation function to be of a given degree. Several results are provided for different degree constraints. These results offer feasibility tests via linear matrix inequalities. We have identified a number of degree constraints for which the feasibility tests are exact. For other degree constraints, we offer a relaxation scheme for checking the feasibility. The second type of constraints we study is about spectral zero assignment, which demands the zeros of the spectral factorization of the interpolation function to be at given locations. This problem can be solved using an iterative algorithm by Byrnes, Georgiou and Linquist. However, we provide a much faster iterative algorithm for this problem, although a proof of convergence is yet to be offered.
\end{abstract}

\section{INTRODUCTION}

This paper is concerned with some generalizations of the following well-known Nevanlinna-Pick interpolation problem: Given $m+1$ distinct complex numbers $\xi_{0}, \xi_{1}, \xi_{1}, \ldots, \xi_{m}$ with $\xi_{0}=\infty$ and $\left|\xi_{k}\right|>1$ for $k=1,2, \cdots, m$, and $m+1$ complex numbers $\hat{f}_{0}, \hat{f}_{1}, \ldots, \hat{f}_{m}$ with $\hat{f}_{k}+\hat{f}_{k}^{*}>0$, find a rational function $f(z)$ such that

$$
f\left(\xi_{k}\right)=\hat{f}_{k}, k=0,1, \ldots, m,
$$

and that $f(z)$ is strictly positive real (SPR), i.e., $f(z)$ is analytic in $|z| \leq 1$ and that

$$
f(z)+f^{*}\left(z^{-1}\right)>0, \quad \forall|z|=1
$$

In the above, the choice of $\xi_{0}=\infty$ is for convenience.

The solutions to the rational interpolation problem are known as Carathéodory functions in the mathematical literature [1] and have vast applications in circuits and systems [2], stability analysis, linear and nonlinear control design [3], signal processing [4], and stochastic systems theory [5].

In most applications, it is important that the interpolating function satisfies additional constraints. In this paper, we consider two important constraints. The first one is a degree constraint, i.e., $f(z)$ is required to be of a given degree $n \leq m$. The second constraint is to assign the spectral zeros of $f(z)$. More precisely, let $\phi(z)$ be the unique spectral factorization of $f(z)$, i.e., $\phi(z)$ is stable with minimum phase and satisfying

$$
f(z)+f^{*}\left(z^{-1}\right)=\phi(z) \phi^{*}\left(z^{-1}\right)
$$

This work is supported by Australian research council

The authors are with the Centre for Complex Dynamic Systems and Control, School of Electrical Engineering and Computer Science, University of Newcastle, Callaghan, NSW 2308, Australia. Minyue.Fu@newcastle.edu.au, Kaushik. Mahata@newcastle.edu.au
Then, the zeros of $\phi(z)$, which are referred to the spectral zeros of $f(z)$, need to be assigned at given locations. This interpolation problem is of interest in spectrum analysis applications [6].

The interpolation problem without degree constraints is a classic problem and has been studied in depth (see a summary in [2]). In particular, a solution is known to exist if the so-called Pick matrix, defined by the given data, is positive definite. In this case, there always exist solutions with degree $m$ [2]. However, when either of the two constraints is present, the interpolation problem becomes much harder. While the Nevanlinna-Schur recursion algorithm [2] and the well-known linear fractional parametrization of all solutions with degree constraints given in [7] can be used to generate rational solutions, they do not provide much clue about how to handle additional constraints. In general, even if the Nevanlinna-Pick problem without degree constraints is solvable, the set of interpolation functions of degree $n<m$ may be empty, and to determine whether this is the case is known to be a hard problem [8]. In contrast for the case $m=n$, it has been shown [8] that solutions to the Nevanlinna-Pick problem can be parameterized using the spectral zeros of the interpolation function. That is, for each set of $n$ stable spectral zeros, there exists a unique solution to the interpolation problem which matches the spectral zeros. In addition, [8] provides an ingenious iterative algorithm for searching the solution.

In this paper, a number of new results are presented for the aforementioned generalization problems. For the degree constraint problem, we first establish necessary and sufficient conditions for testing whether a solution to the NevanlinnaPick problem exists with degree $n=m / 2$ (for even $m$ ) and $n=m 1$. This is followed by a relaxation scheme which gives a sufficient condition for testing the existence of a solution with any given $m / 2<n<m-1$. All the tests are based on linear matrix inequalities (LMIs) and are thus computationally tractable. For the spectral zero assignment problem, we propose a new iterative algorithm much faster than the algorithm in [8]. In fact, the algorithm in [8] requires solving an algebraic Riccati equation in each iteration, whereas our algorithm amounts solving a Lyapunov equation in each iteration. However, we have not established theoretically our algorithm always converges to the desired solution, although the convergence has been tested extensively in simulations.

For notational simplicity, we assume that the sets $\{\xi\}_{i=1}^{n}$ and $\left\{\hat{f}_{i}\right\}_{k=1}^{n}$ are self conjugate, and $\hat{f}_{0}$ is real valued. But all the analysis in the paper can be extended readily even when the assumption above does not hold. 


\section{Degree-Constrained NeVAnlinna-Pick PROBLEM}

In this section, we consider the degree-constrained Nevanlinna-Pick problem for different values of $n$.

The case of $n=m$ corresponds to the classical Neveanlinna-Pick problem without degree constraints and its solvability is determined by the positive definiteness of a corresponding Pick matrix. If the Pick matrix is positive definite, the solution is not unique and can be parameterized in terms of $n$ free parameters [2], [3], [8]. Another case of special interest is $m=2 n$. In this case, the solution to $f(z)$, if exists, is generically unique because $f(z)$ has $2 n+1$ coefficients and there are $m+1$ interpolation points. We will give a necessary and sufficient condition for solving the interpolation problem. We will also study the intermediate situation when $n<m<2 n$ and provide a necessary and sufficient condition for solving the interpolation problem. This condition, however, is difficult to test because it involves a nonlinear matrix inequality. However, for the case of $m=n+1$, we can replace this condition with a LMI without conservatism. For $n+1<m<2 n$, we offer a relaxation scheme using a LMI.

We start by introducing a simple characterization of all possible rational functions which satisfy the first $n$ interpolation data and $f(\infty)=\hat{f}_{0}$ but not necessarily the SPR condition. This will help simplify calculations to a significant extent and give us an additional insight into the interpolation problem.

Lemma 1: Let us define the $n \times n$ diagonal matrix $\Omega, n \times 1$ vectors $w$ and $\hat{f}$ as

$$
\Omega=\left[\begin{array}{ccc}
\xi_{1} & \cdots & 0 \\
\vdots & \ddots & \vdots \\
0 & \cdots & \xi_{n}
\end{array}\right], w=\left[\begin{array}{c}
1 \\
\vdots \\
1
\end{array}\right], \hat{f}=\left[\begin{array}{c}
\hat{f}_{1} \\
\vdots \\
\hat{f}_{n}
\end{array}\right]
$$

Let $a$ be any $n \times 1$ vector. Then

$$
f(z)=\frac{\hat{f}_{0}+a^{*}\left(z I_{n}-\Omega\right)^{-1} \hat{f}}{1+a^{*}\left(z I_{n}-\Omega\right)^{-1} w}
$$

satisfy the interpolation conditions in (1) for $k=1, \ldots, n$ and $f(\infty)=\hat{f}_{0}$.

Proof: The proof simply follows by taking $z \rightarrow \xi_{k}$ in (4) for each $k$ or $z \rightarrow \infty$ and evaluating the limit.

Using the lemma above we can parameterize all the degree- $n$ rational functions satisfying (1) via the free parameter vector $a$. As $a$ determines the poles of the representation, we see that for any choice of the poles of the rational representation there exists a unique $a$ and thus a unique $f(z)$ such that the first $n$ interpolation conditions in (1) are satisfied. However, not every a makes $f(z)$ satisfy the SPR condition (2).

\section{A. The case $m=n$}

In this case, the remaining $n$ degrees of freedom can be used to satisfy additional constraints. In [8], [9], it was shown that it is possible to specify $n$ spectral zeros, provided that they are inside the closed unit disc. Therefore, the solution set is completely parameterized via spectral zeros. In what follows next, we seek for an alternative parameterization of the solution set when $m=n$.

Lemma 2: The rational function $f(z)$ in (4) is SPR if and only if there exists a $n \times n$ Hermitian positive definite matrix $Q$ and a $n \times 1$ vector $a_{\star}$ to satisfy the following LMI:

$$
\left[\begin{array}{ccc}
\hat{f} w^{*}+w \hat{f}^{*}+Q & \hat{f}+w \hat{f}_{0} & \Omega Q \\
\hat{f}_{0} w^{*}+\hat{f}^{*} & 2 \hat{f}_{0} & a_{\star}^{*} \\
Q \Omega^{*} & a_{\star} & Q
\end{array}\right]>0
$$

When the above holds, $a$ is related to $Q$ and $a_{\star}$ via

$$
a=Q a_{\star}
$$

Proof: Let us denote the numerator in (4) by $\Gamma(z)$ and the denominator in (4) by $\Delta(z)$. Since we assume that the sets $\{\xi\}_{i=1}^{n}$ and $\left\{\hat{f}_{i}\right\}_{k=1}^{n}$ are self-conjugate, we can write

$$
\begin{aligned}
\Gamma(z) & =\left[\begin{array}{ll}
\hat{f}^{*} & \hat{f}_{0}
\end{array}\right]\left[\begin{array}{c}
\left(z I_{n}-\Omega^{*}\right)^{-1} a \\
1
\end{array}\right], \\
\Delta(z) & =\left[\begin{array}{ll}
w^{*} & 1
\end{array}\right]\left[\begin{array}{c}
\left(z I_{n}-\Omega^{*}\right)^{-1} a \\
1
\end{array}\right] .
\end{aligned}
$$

Then $f(z)$ is SPR if and only if

$$
\begin{gathered}
\Gamma(z) \Delta^{*}\left(z^{-1}\right)+\Delta(z)+\Gamma^{*}\left(z^{-1}\right)=\left[\begin{array}{c}
\left(z^{-1} I_{n}-\Omega^{*}\right)^{-1} a \\
1
\end{array}\right]^{*} \\
\times\left[\begin{array}{cc}
\hat{f} w^{*}+w \hat{f}^{*} & \hat{f}+w \hat{f}_{0} \\
\hat{f}_{0} w^{*}+\hat{f}^{*} & 2 \hat{f}_{0}
\end{array}\right]\left[\begin{array}{c}
\left(z I_{n}-\Omega^{*}\right)^{-1} a \\
1
\end{array}\right]
\end{gathered}
$$

is SPR. Next, we apply Kalman-Yakubovitch-Popov lemma [10], [11] to find SPR condition. The function $f(z)$ in (4) is SPR if and only if there exists a $n \times n$ Hermitian positive definite matrix $Q$ such that

$$
\left[\begin{array}{cc}
\hat{f} w^{*}+w \hat{f}^{*}+Q & \hat{f}+w \hat{f}_{0} \\
\hat{f}_{0} w^{*}+\hat{f}^{*} & 2 \hat{f}_{0}
\end{array}\right]>\left[\begin{array}{c}
\Omega \\
a^{*}
\end{array}\right] Q\left[\begin{array}{ll}
\Omega^{*} & a
\end{array}\right]
$$

Now setting $Q=Q Q^{-1} Q$ in the right hand side of (7), using (6) and taking Schur complement, we obtain (5).

Lemma 2 provides us with an alternative approach to parameterizing all the solutions to the Nevanlinna-Pick problem via the LMI (5) in $Q$ and $a_{\star}$. Each feasible solution $\left(Q, a_{\star}\right)$ then leads us to a feasible $a$. However it is interesting to investigate the conditions on the given data in order to guarantee that such a feasible $a$ exists. This is established in the next Theorem.

Theorem 1: Let us define $\Psi=\hat{f} w^{*}+w \hat{f}^{*}$. Then there exist $a_{\star}$ and $Q$ (Hermitian and positive definite) such that (5) holds if and only if the following LMIs

$$
\Omega Q \Omega^{*}-Q<\Psi,\left[\begin{array}{cc}
Q+\Psi & \hat{f}+w \hat{f}_{0} \\
\hat{f}^{*}+\hat{f}_{0} w^{*} & 2 \hat{f}_{0}
\end{array}\right]>0
$$

hold for the same $Q$.

Proof: The LMI (5) can be written as

$$
R+U a_{\star} V^{\top}+V a_{\star} U^{\top}>0
$$


where

$$
\begin{gathered}
R=\left[\begin{array}{ccc}
Q+\Psi & \hat{f}+w \hat{f}_{0} & \Omega Q \\
\hat{f}_{0} w^{*}+\hat{f}^{*} & 2 \hat{f}_{0} & 0_{1 \times n} \\
Q \Omega^{*} & 0_{n \times 1} & Q
\end{array}\right], \\
U=\left[\begin{array}{c}
0_{n \times n} \\
0_{1 \times n} \\
I_{n}
\end{array}\right], \quad V=\left[\begin{array}{c}
0_{n \times 1} \\
1 \\
0_{n \times 1}
\end{array}\right] .
\end{gathered}
$$

It is straightforward to construct the full-rank matrices $U_{\perp}$ and $V_{\perp}$ orthogonal to $U$ and $v$, respectively:

$$
V_{\perp}=\left[\begin{array}{cc}
I_{n} & 0_{n \times n} \\
0_{1 \times n} & 0_{1 \times n} \\
0_{n \times n} & I_{n}
\end{array}\right], U_{\perp}=\left[\begin{array}{c}
I_{2 n} \\
0_{n \times 2 n}
\end{array}\right]
$$

Now by the elimination lemma [11], [12], there exists $a_{\star}$ such that (5) holds if and only if $U_{\perp}^{\top} R U_{\perp}>0$ and $V_{\perp}^{\top} R V_{\perp}>0$. The first condition gives the second inequality in (8). From the second condition we get

$$
\left[\begin{array}{cc}
\Psi+Q & \Omega Q \\
Q \Omega^{*} & Q
\end{array}\right]>0
$$

which is converted into (8) by using Schur complement.

Remark: The results in Theorem 1 are similar to the results derived in [12] for the so-called continuous-time bounded-real interpolation problem. However, the results here are slightly more general as pointed out shortly. We also emphasize that our proof is simpler that that in [12].

Next, we want to find out conditions on the interpolation data under which (8) holds. It is natural to expect that this condition is related to the Pick matrix. When $\xi_{0}$ is finite, the Pick matrix is defined as

$$
\bar{P}=\left[\frac{\hat{f}_{i}+\hat{f}_{j}^{*}}{\xi_{i} \xi_{j}^{*}-1}\right]_{i, j=0}^{m}
$$

However, since $\xi_{0}=\infty$, the first row and column of $\bar{P}$ vanish, which makes $\bar{P}$ unusable. To rectify this problem, we return to the case when $\xi_{0}$ is finite and note that $\bar{P}>0$ if and only if the matrix below is positive definite:

$$
\hat{P}=\left[\frac{\xi_{i} \xi_{j}^{*}}{\xi_{i} \xi_{j}^{*}-1}\left(\hat{f}_{i}+\hat{f}_{j}^{*}\right)\right]_{i, j=0}^{m}
$$

which is obtained by multiplying $\operatorname{diag}\left\{\xi_{0}, \xi_{1}, \ldots, \xi_{m}\right\}$ and its Hermitian to the left and right of $\bar{P}$, respectively. Now, taking $\xi_{0} \rightarrow \infty$, the first row and column of $\hat{P}$ no longer vanish. By moving the first row and column to the last, $\hat{P}$ becomes the following modified Pick matrix:

$$
\tilde{P}:=\left[\begin{array}{cc}
\Omega \bar{P}_{m} \Omega^{*} & \hat{f}+w \hat{f}_{0} \\
\hat{f}_{0} w^{*}+\hat{f}^{*} & 2 \hat{f}_{0}
\end{array}\right] .
$$

where $\bar{P}_{m}$ is $\bar{P}$ without the first row and column.

The expected result is given below.

Corollary 2: The LMI conditions given by (8) are equivalent to $\tilde{P}$ being positive definite.
Proof: First note from the definition of $\bar{P}_{m}$ and $\Psi$ that

$\Omega \bar{P}_{m} \Omega^{*}=\Psi+\bar{P}_{m} \Rightarrow\left[\begin{array}{cc}\bar{P}_{m}+\Psi & \hat{f}+w \hat{f}_{0} \\ \hat{f}^{*}+\hat{f}_{0} w^{*} & 2 \hat{f}_{0}\end{array}\right]=\tilde{P}$.

First we show the sufficiency. Suppose that $\tilde{P}$ is positive definite. This implies $\bar{P}_{m}$ is positive definite. Now set $Q=\bar{P}_{m}$ in (8). Comparing with (13) we see that the first inequality in (8) becomes an equality if we have $Q=\bar{P}_{m}$, while the second inequality is satisfied. Therefore, it is possible to find $Q>0$ (by perturbing from $\bar{P}_{m}$ a little) such that both the inequalities in (8) hold.

Conversely, suppose that there exists $Q>0$ such that the LMIs in (8) hold. Then using the first equality in (13) in the first inequality in (8) we get

$$
\Omega\left(\bar{P}_{m}-Q\right) \Omega^{*}>\bar{P}_{m}-Q \Rightarrow \bar{P}_{m}>Q
$$

since $\left|\xi_{i}\right|>1$ for all $i$. Now we use the second equality in (13) in the second inequality of (8). We get

$$
\tilde{P}>\left[\begin{array}{cc}
\bar{P}_{m}-Q & 0_{m \times 1} \\
0_{1 \times m} & 0
\end{array}\right] \geq 0,
$$

which follows from (14), and the result is proven.

\section{B. The case $m>n$}

If $f(z)$ in (4) satisfies the interpolation constraints in (1), then it follows that

$$
\left(\hat{f}-w \hat{f}_{k}\right)^{*}\left(\xi_{k}^{*} I_{n}-\Omega^{*}\right)^{-1} a=\hat{f}_{k}^{*}-\hat{f}_{0}
$$

for $1 \leq k \leq m$. If we have $m>n$, the equation above serves as additional linear constraints on the parameter vector $a$, and thereby reducing the degree of freedom. We can form a linear equality constraint in the matrix form as

$$
\check{f}=G a
$$

where

$$
\begin{aligned}
\check{f}= & {\left[\begin{array}{lll}
\hat{f}_{n+1}^{*}-\hat{f}_{0} & \cdots & \hat{f}_{m}^{*}-\hat{f}_{0}
\end{array}\right]^{\top}, } \\
G= & {\left[\begin{array}{c}
\left(\hat{f}-w \hat{f}_{n+1}\right)^{*}\left(\xi_{n+1}^{*} I_{n}-\Omega^{*}\right)^{-1} \\
\vdots \\
\left(\hat{f}-w \hat{f}_{m}\right)^{*}\left(\xi_{m}^{*} I_{n}-\Omega^{*}\right)^{-1}
\end{array}\right] }
\end{aligned}
$$

In the rest of this section, we assume, without loss of generality, that $G$ has a full row rank. Indeed, if $G$ has rank deficiency, some of the interpolation points are redundant and can be removed.

The general solution for $a$ can then be written as

$$
a=a_{0}+G_{\perp}^{*} \alpha, \quad a_{0}=G^{*}\left[G G^{*}\right]^{-1} \check{f},
$$

where $G_{\perp}$ is a $n \times(2 n-m)$ matrix having a full column rank such that $G G_{\perp}=0$. For the special case $m=2 n$, the term $G_{\perp}^{*} \alpha$ vanishes and $a$ is completely known, i.e., $a=a_{0}=G^{-1} \check{f}$. It turns out that we can check whether this unique solution is SPR using an LMI. This is formally stated in the following theorem. 
Theorem 3: Suppose $n=2 m$ and $G$ in (15) is invertible. Then the interpolation problem has a unique solution given by (4) with $a=G^{-1} \check{f}$. This solution is SPR if and only if there exists a Hermitian and positive definite $Q$ to satisfy the following LMI:

$\left[\begin{array}{cc}Q+\Psi & \hat{f}+w \hat{f}_{0} \\ \hat{f}_{0} w^{*}+\hat{f}^{*} & 2 \hat{f}_{0}\end{array}\right]>\left[\begin{array}{c}\Omega \\ \check{f}^{*} G^{-*}\end{array}\right] Q\left[\begin{array}{ll}\Omega^{*} & G^{-1} \check{f}\end{array}\right]$

Proof: The solution to $a$ follows from the discussion prior to the theorem and the LMI condition follows from Lemma 2 (see (7) in particular).

Next we investigate the case when $n<m<2 n$. Naturally, in this case the solvability conditions become more stringent as we increase $m$. In the following lemma we formulate the solvability conditions.

Lemma 3: Suppose $n<m<2 n$. Then there exists $\alpha$ as in (16) such that $f(z)$ in (4) is SPR if and only if

$$
\Omega Q \Omega^{*}-Q<\Psi
$$

and

$$
\left[\begin{array}{ccc}
Q+\Psi & \hat{f}+w \hat{f}_{0} & \Omega G^{*} \\
\hat{f}_{0} w^{*}+\hat{f}^{*} & 2 \hat{f}_{0} & \check{f}^{*} \\
G \Omega^{*} & \check{f} & G Q^{-1} G^{*}
\end{array}\right]>0
$$

for some Hermitian and positive definite $Q$.

Proof: Taking Schur complement in (7) and using (16) we see that $f(z)$ in (4) is SPR if and only if

$$
\left[\begin{array}{ccc}
Q+\Psi & \hat{f}+w \hat{f}_{0} & \Omega \\
\hat{f}_{0} w^{*}+\hat{f}^{*} & 2 \hat{f}_{0} & a_{0}^{*} \\
\Omega^{*} & a_{0} & Q^{-1}
\end{array}\right]+\bar{U} \alpha V^{*}+V \alpha^{*} \bar{U}^{*}>0
$$

for some positive definite $Q$ and $\alpha$. Here we have used

$$
\bar{U}=\left[\begin{array}{c}
0_{(n+1) \times(2 n-m)} \\
G_{\perp}
\end{array}\right] .
$$

and $V$ is as in the proof of Theorem 1. We have

$$
\bar{U}_{\perp}=\left[\begin{array}{cc}
I_{2 n+1} & 0_{(2 n+1) \times(m-n)} \\
0_{(m-n) \times(2 n+1)} & G
\end{array}\right] .
$$

By the elimination lemma, there exists $\alpha$ and $Q$ such that (19) is satisfied if and only if (9) and (18) hold. Finally, it is easy to verify that (17) is equivalent to (9).

It is interesting to see how the additional interpolation constraints impose more and more stringent requirements for solvability of the problem. Comparing (18) with the second inequality in (8) we see that when $m=n$ we only need to satisfy the inequality obtained by taking the first $n+1$ rows and first $n+1$ columns of (18). The $(n+1)$ th interpolation condition then adds the $(n+2)$-th row and the $(n+2)$-th column in (18), and the process continues. It is straightforward to see that the solvability for $n+k$ interpolation constraints implies solvability for $n+k-1$ interpolation conditions, but converse is not true. Finally, when $m=2 n$, the feasible set contain a maximum of one point.

It is evident that (18) gives a non-convex set in $Q$, and it is in general difficult to solve (18) using any numerical technique. For this purpose we propose a relaxation scheme in the following. The idea of the relaxation scheme is to find a convex subset of the set of feasible $Q$ given by (18).

Theorem 4: Consider the case when $n<m<2 n$. Then there exists a solution to the interpolation problem if the LMI

$$
\left[\begin{array}{cc}
Q+\Psi & \hat{f}+w \hat{f}_{0} \\
\hat{f}_{0} w^{*}+\hat{f}^{*} & 2 \hat{f}_{0}
\end{array}\right]>\left[\begin{array}{c}
\Omega \Pi_{G} \\
\check{f}^{*} G^{\dagger *}
\end{array}\right] Q\left[\begin{array}{ll}
\Pi_{G} \Omega^{*} & G^{\dagger} \check{f}
\end{array}\right]
$$

is feasible in a positive definite matrix $Q$, where

$$
G^{\dagger}=G^{*}\left(G G^{*}\right)^{-1}, \quad \Pi_{G}=G^{\dagger} G .
$$

Proof: We first note that feasibility of the LMI

$\left[\begin{array}{cccc}Q+\Psi & \hat{f}+w \hat{f}_{0} & \Omega G^{*} & 0_{n \times(2 n-m)} \\ \hat{f}_{0} w^{*}+\hat{f}^{*} & 2 \hat{f}_{0} & \check{f}^{*} & 0_{1 \times(2 n-m)} \\ G \Omega^{*} & \check{f} & G Q^{-1} G^{*} & G Q^{-1} G_{\perp} \\ 0_{(2 n-m) \times n} & 0_{(2 n-m) \times 1} & G_{\perp}^{*} Q^{-1} G^{*} & G_{\perp}^{*} Q^{-1} G_{\perp}\end{array}\right]>0$

implies (18). However, the converse is not true. Now taking Schur complement of (21) we have

$$
\begin{aligned}
& {\left[\begin{array}{cc}
Q+\Psi & \hat{f}+w \hat{f}_{0} \\
\hat{f}_{0} w^{*}+\hat{f}^{*} & 2 \hat{f}_{0}
\end{array}\right]>\left[\begin{array}{cc}
\Omega G^{*} & 0_{n \times(2 n-m)} \\
\check{f}^{*} & 0_{1 \times(2 n-m)}
\end{array}\right] \times} \\
& {\left[\begin{array}{ll}
G^{*} & G_{\perp}
\end{array}\right]^{-1} Q\left[\begin{array}{c}
G \\
G_{\perp}^{*}
\end{array}\right]^{-1}\left[\begin{array}{cc}
\Omega G^{*} & 0_{n \times(2 n-m)} \\
\check{f}^{*} & 0_{1 \times(2 n-m)}
\end{array}\right]^{*}}
\end{aligned}
$$

Now note that

$$
\left[\begin{array}{c}
G \\
G_{\perp}^{*}
\end{array}\right]^{-1}=\left[\begin{array}{ll}
G^{\dagger} & G_{\perp}^{\dagger *}
\end{array}\right]
$$

which we substitute in (22) to get (20).

Next we study the case $m=n+1$. Our goal is to show that we do not need any relaxation in this case, i.e. we can get a necessary and sufficient condition for the solvability.

Theorem 5: Suppose $m=n+1$. Then there exists a solution to the interpolation problem if and only if

$$
\lambda_{\star} \leq G Q^{-1} G^{*}
$$

where $\lambda_{\star}$ and $Q$ (Hermitian and positive definite) are the solution to the optimization problem

$$
\begin{array}{clcc} 
& \lambda_{\star}=\inf \lambda, \\
\text { subject to } & {\left[\begin{array}{ccc}
Q+\Psi & \hat{f}+w \hat{f}_{0} & \Omega G^{*} \\
\hat{f}_{0} w^{*}+\hat{f}^{*} & 2 \hat{f}_{0} & \check{f}^{*} \\
G \Omega^{*} & \check{f} & \lambda
\end{array}\right]>0,}
\end{array}
$$

Proof: For the case $m=n+1$, the term $G Q^{-1} G^{*}$ in (18) is a scalar. We denote

$$
\alpha(Q):=\left[\begin{array}{ll}
G \Omega^{*} & \check{f}
\end{array}\right] \Pi(Q)\left[\begin{array}{c}
\Omega G^{*} \\
\check{f}^{*}
\end{array}\right]
$$

with

$$
\Pi(Q)=\left[\begin{array}{cc}
Q+\Psi & \hat{f}+w \hat{f}_{0} \\
\hat{f}_{0} w^{*}+\hat{f}^{*} & 2 \hat{f}_{0}
\end{array}\right]
$$


Then, the optimal solution $\lambda_{\star}$ can be rewritten as

$$
\lambda_{\star}=\inf _{Q} \alpha(Q), \text { subject to } \Omega Q \Omega^{*}-Q<\Psi
$$

From Lemma 3, the interpolation problem admits a solution if and only if there exists $Q=Q^{*}>0$ such that

$$
G Q^{-1} G^{*}>\alpha(Q) ; \quad \Omega Q \Omega^{*}-Q<\Psi
$$

If (23) holds for some $\lambda_{\star}$ and $Q_{\star}$, then by (24) we have $G Q_{\star}^{-1} G^{*}>\lambda_{\star}=\alpha\left(Q_{\star}\right)$ which implies (25) with $Q=Q_{\star}$. The proof of the converse is more involved and thus omitted.

\section{SPECTRAL ZERO ASSIGNMENT}

In this section we reconsider the case $m=n$. Here our objective is to derive a fast algorithm for the Nevanlinna-Pick interpolation problem subject to spectral zero assignment. To this end, we first recite a key result in [8], [9].

Theorem 6: Let the modified Pick matrix $\tilde{P}$ in (12) be positive definite and $n=m$. Then for every monic marginally Schur stable polynomial $\bar{\sigma}(z)$ of order $n$, there exists a unique $n$-th order monic Schur stable polynomial $\bar{\tau}(z)$ such that

$$
f(z)+f^{*}\left(z^{-1}\right)=\rho^{2} \frac{\bar{\sigma}^{*}\left(z^{-1}\right)}{\bar{\tau}^{*}\left(z^{-1}\right)} \frac{\bar{\sigma}(z)}{\bar{\tau}(z)}
$$

such that the interpolation conditions in (1) are satisfied, where the gain $\rho$ is determined by $f(\infty)=\hat{f}_{0}$.

Proof: When $\xi_{0}$ is finite, the result above comes from [8], [9]. When taking $\xi_{0} \rightarrow \infty$, the result remains except that the modified Pick matrix $\tilde{P}$ is used.

In contrast to the previous section we take a different approach here. In the previous section we formulated a state space realization of $f(z)$ such that the interpolation conditions in (1) are automatically satisfied. Here we work with a different realization, where we parameterize the minimum phase spectral factor $\bar{\sigma}(z) / \bar{\tau}(z)$ of $f(z)+f^{*}\left(z^{-1}\right)$ such that the spectral zeros are automatically assigned to the desired points. Now the free parameters are the coefficients of denominator polynomial $\bar{\tau}(z)$. Hence it remains to tune only the coefficients of $\bar{\tau}(z)$ so that the interpolation conditions in (1) are satisfied while maintaining the SPR condition.

We express

$$
\begin{aligned}
& \bar{\sigma}(z)=z^{n}+\sigma_{1} z^{n-1}+\cdots+\sigma_{n}, \\
& \bar{\tau}(z)=z^{n}+\tau_{1} z^{n-1}+\cdots+\tau_{n} .
\end{aligned}
$$

and denote

$$
\begin{gathered}
\sigma=\left[\begin{array}{ccc}
\sigma_{1} & \cdots & \sigma_{n},
\end{array}\right]^{\top} ; \tau=\left[\begin{array}{ccc}
\tau_{1} & \cdots & \tau_{n}
\end{array}\right]^{\top} \\
J=\left[\begin{array}{cccc}
0 & 1 & \cdots & 0 \\
\vdots & \vdots & \ddots & \vdots \\
0 & 0 & \cdots & 1 \\
0 & 0 & \cdots & 0
\end{array}\right], h=\left[\begin{array}{c}
1 \\
0 \\
\vdots \\
0
\end{array}\right], F=J-\tau h^{\top} .
\end{gathered}
$$

Hence the problem under consideration is to find $\tau$ such that the interpolation conditions in (1) are satisfied. Using the observable canonical state-space realization, we have

$$
\frac{\bar{\sigma}(z)}{\bar{\tau}(z)}=1+h^{\top}\left[z I_{n}-F\right]^{-1}(\sigma-\tau) .
$$

Then $f(z)$ in (26) is uniquely given by [5]

$f(z)=\frac{\rho^{2}}{2}\left(1+h^{\top} P h\right)+\rho^{2} h^{\top}[z I-F]^{-1}\{F P h+(\sigma-\tau)\}$,

where $P$ is a symmetric and positive definite matrix satisfying

$$
P=F P F^{\top}+(\sigma-\tau)(\sigma-\tau)^{\top} .
$$

We are ready to state the following lemma.

Lemma 4: Let us define

$$
\begin{gathered}
D_{1}=\operatorname{diag}\left\{\begin{array}{ccc}
\frac{\hat{f}_{1}-\hat{f}_{0}}{\hat{f}_{1}+\hat{f}_{0}} & \cdots & \frac{\hat{f}_{n}-\hat{f}_{0}}{\hat{f}_{n}+\hat{f}_{0}}
\end{array}\right\}, \\
D_{2}=\operatorname{diag}\left\{\begin{array}{ccc}
\frac{1}{\hat{f}_{1}+\hat{f}_{0}} & \cdots & \frac{1}{\hat{f}_{n}+\hat{f}_{0}}
\end{array}\right\}, \\
L=\left[\begin{array}{cccc}
\xi_{1}^{n-1} & \cdots & \xi_{1} & 1 \\
\vdots & \ddots & \vdots & \vdots \\
\xi_{n}^{n-1} & \cdots & \xi_{n} & 1
\end{array}\right], \ell=\left[\begin{array}{c}
\xi_{1}^{n} \\
\vdots \\
\xi_{n}^{n}
\end{array}\right],
\end{gathered}
$$

and

$$
x=L^{-1} D_{1} \ell, \quad X=L^{-1} D_{2} L .
$$

Then $f(z)$ in (28) satisfies the interpolation conditions in (1) if and only if

$$
\tau=\frac{2 \hat{f}_{0}}{1+h^{\top} P h} X(J P h+\sigma)-x,
$$

and

$$
\rho^{2}=\frac{2 \hat{f}_{0}}{1+h^{\top} P h} .
$$

where $P$ satisfies (29).

Proof: Taking $z \rightarrow \infty$ in (28) we get

$$
f(\infty)=\frac{\rho^{2}}{2}\left(1+h^{\top} P h\right) .
$$

Recall that $\xi_{0}=\infty$. Thus, the interpolation condition at $\xi_{0}$ gives (31). It is easy to verify that

$$
h^{\top}[z I-F]^{-1}=\frac{q(z)}{z^{n}+q(z) \tau},
$$

where

$$
q(z)=\left[\begin{array}{llll}
z^{n-1} & \cdots & z & 1
\end{array}\right] .
$$

Now by combining the above observations, we get

$$
\frac{f(z)-f(\infty)}{\rho^{2}}\left[z^{n}+q(z) \tau\right]=q(z)\left[J-\tau h^{\top}\right] P h+q(z)(\sigma-\tau) .
$$

Using (32) and rearranging the equation above, we get

$$
\frac{f(z)-f(\infty)}{f(z)+f(\infty)} z^{n}+q(z) \tau=\frac{\rho^{2} q(z)}{f(z)+f(\infty)}(J P h+\sigma) .
$$


Now we apply the interpolation constraints in (1). For each interpolation point, (33) gives an equation. Stacking these equations in a matrix form we get

$$
D_{1} \ell+L \tau=\rho^{2} D_{2} L(J P h+\sigma),
$$

which after a rearrangement using (31) gives (30).

Lemma 4 gives us a way to find $\tau$. From (29) we can find $P$ as a function of $\tau$ by solving (29). But (30) gives $\tau$ as a function of $P$. Therefore, we can eliminate $\tau$ to obtain a generalized Riccati equation in $P$. However, it is not clear how to solve it. Instead we can solve the equation using (29) and (30) repeatedly until convergence. The Recursion is as follows. Initialize $k=0$ and $P_{0}=I_{n}$. Then the following steps are repeated until convergence:

1) $\rho_{k}^{2}=\frac{2 f(\infty)}{1+h^{\top} P_{k} h}$

2) $\tau^{(k)}=\rho_{k}^{2} X\left(J P_{k} h+\sigma\right)-x$,

3) $F_{k}=J-\tau^{(k)} h^{\top}$

4) $P_{k+1}=F_{k} P_{k} F_{k}+\left(\sigma-\tau^{(k)}\right)\left(\sigma-\tau^{(k)}\right)^{\top}$

Although we do not have a theoretical justification that the recursion described above converges, extensive numerical study has shown that the recursion does always converge. It is also interesting to compare the above recursive algorithm with the Byrnes-Georgiou-Lindquist algorithm proposed in [8]. In [8], a gradient decent method is used to compute the solution. However, each iteration of the gradient decent amounts to solving a Riccati equation and searching for an optimal step size. The proposed algorithm involves solving a Lyapunov equation (Step 4) plus some minor computations (Steps 1, 2 and 3). This makes the proposed algorithm much more efficient from a practical point of view.

\section{Simulation Results}

We consider the results in Section II in a numerical simulation study by considering interpolation problems of degree 2,46 and 8, respectively. For each degree $n$ we consider a known positive real transfer function $g(z)$, which is used to generate the interpolation data by $\hat{f}_{i}=g\left(\xi_{i}\right)$. In this way we can guarantee that the interpolation problem considered in the simulation experiment always has a solution. The main idea is to test the relaxation scheme described via Theorem 4. For each value of $n$, we consider the cases $m=n$, $n<m<2 n$ and $m=2 n$. For each pair $(m, n)$ we consider 100 different interpolation problems corresponding to 100 different combinations of $\left\{\xi_{i}\right\}_{i=1}^{m}$ chosen randomly outside the unit disc. It is expected that when $m=n$ we should always get a feasible solution by using Theorem 1 . Similarly, the unique solution (which should be $g(z)$ ) for the case $m=2 n$ should be tested to be a positive real solution by using the result in Theorem 3. However, for $n<m<2 n$, simulation results are used to check the tightness of the relaxation scheme in Theorem 4. Simulation results are shown in Table I, where the number in each box indicates the success rate (in percentage). We notice that the relaxation is reasonably tight when the degree is low.

\begin{tabular}{|c|c|c|c|c|c|c|c|c|}
\hline $\mathrm{m}$ & 3 & 4 & 6 & 8 & 10 & 12 & 14 & 16 \\
\hline \hline$n=2$ & 100 & 100 & - & - & - & - & - & - \\
\hline$n=4$ & - & 100 & 100 & 100 & - & - & - & - \\
\hline$n=6$ & - & - & 100 & 100 & 93 & 100 & - & - \\
\hline$n=8$ & - & - & - & 100 & 99 & 73 & 42 & 100 \\
\hline
\end{tabular}

TABLE I

However, as the degree increases, we get a lower percentage of correct outcomes.

\section{CONCLUSION}

We have provided a number of new results on constrained Nevanlinna-Pick interpolation problems. The results on degree constrained problems are derived based on a new parameterization of the unconstrained solution set, as given in Lemmas 1 and 2. This leads to a number of LMI based tests for the solvability of degree constrained interpolation problem. For the cases of $m=2 n, m=n$ and $m=n+1$, the tests are exact. For the case of $n+1<m<2 n$, it would be interesting to see whether the relaxation scheme can be further improved. For the spectral zero assignment problem, our solution is based on a totally different parameterization of the unconstrained solution set, as given in Lemma 4. The result is a very fast iterative algorithm. It remains to be verified theoretically that this algorithm always converges correctly, although this is the case in numerous simulations we have conducted so far.

\section{REFERENCES}

[1] N. I. Akhiezer, The classical moment problem. London: Oliver and Boyd, 1965.

[2] P. Delsarte, Y. Genin, and Y. Kamp, "On the role of the NevanlinnaPick problem in circuits and system theory," Circuit Theory and Applicaions, vol. 9, pp. 177-187, 1981.

[3] H. Kimura, "Robust stabilizability for a class of transfer functions," IEEE Transactions on Automatic Control, vol. AC-29, pp. 788-793, September 1984.

[4] T. T. Georgiou, "Realization of power spectra from partial covariance sequences," IEEE Transactions on Acoustics, Speech and Signal Processing, vol. ASSP-35, pp. 438-449, 1987.

[5] C. I. Byrnes and A. Lindquist, "On the partial stochastic realization problem," IEEE Transactions on Automatic Control, vol. 42:8, pp. 1049-1070, August 1997.

[6] C. I. Byrnes, T. T. Georgiou, and A. Lindquist, "A new approach to spectral estimation: A tunable high-resolution spectral estimator," IEEE Transactions on Signal Processing, vol. 48:4, pp. 3189-3205, November 2000.

[7] J. L. Walsh, Interpolation and approximation by rational functions in the complex domain. American Mathematical Society Colloquium Publications, 1956, vol. 20.

[8] C. I. Byrnes, T. T. Georgiou, and A. Lindquist, "A generalized entropy criterion for Nevanlinna-Pick interpolation with degree constraint," IEEE Transactions on Automatic Control, vol. 46:6, pp. 822-839, June 2001.

[9] T. T. Georgiou, "The interpolation problem with a degree constraint," IEEE Transactions on Automatic Control, vol. 44:3, pp. 631-635, March 1999.

[10] A. Rantzer, "On the Kalman-Yakubovitz-Popov lemma," Systems \& Control Letters, vol. 28, pp. 7-10, 1996.

[11] S. Boyd, L. E. Ghaoui, E. Feron, and V. Balakrishnan, Linear Matrix Inequalities in System and Control Theory. SIAM, 1994.

[12] E. van der Meché and O. Bosgra, "Nevanlinna-Pick interpolation with degree constraints: complete characterization based on Lyapunoov inequalities," in 43rd IEEE conference on Decision and Control, Atlantis, Paradise Islands, Bahamas, December 2004, pp. 471-476. 